PROCEEDINGS OF THE AMERICAN MATHEMATICAL SOCIETY

Volume 124, Number 7, July 1996

\title{
THE LU QI-KENG CONJECTURE FAILS GENERICALLY
}

\author{
HAROLD P. BOAS \\ (Communicated by Eric Bedford)
}

\begin{abstract}
The bounded domains of holomorphy in $\mathbf{C}^{n}$ whose Bergman kernel functions are zero-free form a nowhere dense subset (with respect to a variant of the Hausdorff distance) of all bounded domains of holomorphy.
\end{abstract}

A domain in $\mathbf{C}^{n}$ is called a $L u$ Qi-Keng domain if its Bergman kernel function has no zeroes. Lu Qi-Keng [11] raised the question of which domains, besides the ball and the polydisc, have this property. A motivation for the question is that the vanishing of the Bergman kernel function obstructs the global definition of Bergman representative coordinates. Over the years since Lu Qi-Keng's paper appeared, various versions of a $L u Q i$-Keng conjecture have been mooted to the effect that all domains, or most domains, or all domains satisfying some geometrical hypothesis, are Lu Qi-Keng domains.

In the complex plane $\mathbf{C}^{1}$, a bounded domain with smooth boundary is a Lu QiKeng domain if and only if it is simply connected [16] (and thus biholomorphically equivalent to the disc). I have given a counterexample [1] showing that no analogous topological characterization of Lu Qi-Keng domains can hold in higher dimensions: there exists (in $\mathbf{C}^{2}$, and similarly in $\mathbf{C}^{n}$ for $n>2$ ) a bounded, strongly pseudoconvex, contractible domain with $C^{\infty}$ regular boundary whose Bergman kernel function does have zeroes.

In this note, I show that the Lu Qi-Keng domains of holomorphy may be viewed as exceptional: they form a nowhere dense set with respect to a suitable topology. Thus, contrary to former expectations, it is the normal situation for the Bergman kernel function of a domain to have zeroes.

To formulate the result precisely, I need a metric on bounded open sets. Since I impose no restriction on the regularity of the boundaries of the sets, some variant of the Hausdorff metric will be appropriate. The Hausdorff distance $\mathcal{H}$ is normally defined for nonempty, bounded, closed sets by the property that $\mathcal{H}(A, B)<\epsilon$ if and only if each point of $A$ has Euclidean distance less than $\epsilon$ from some point of $B$, and vice versa.

After the seminal paper of Ramadanov [12], it is clear in the context of the Bergman kernel function that if a sequence of open sets $\left\{\Omega_{j}\right\}$ is going to be said to converge to an open set $\Omega$, then every compact subset of $\Omega$ should eventually be contained in $\Omega_{j}$. It is less clear what requirement should be imposed if the $\Omega_{j}$ contain points outside of $\Omega$. The example [15, p. 39], [13, p. 280] of decreasing

Received by the editors December 10, 1994.

1991 Mathematics Subject Classification. Primary 32H10.

This research was partially supported by NSF grant number DMS-9203514.

(c)1996 American Mathematical Society 
concentric disks in the complex plane converging to a disk with a slit removed shows that it is inadequate to require merely that for every open neighborhood of the closure $\bar{\Omega}$, eventually $\Omega_{j}$ is contained in the neighborhood.

I shall consider two different notions of convergence of open sets in $\mathbf{C}^{n}$. Both have the property that if $\Omega_{j} \rightarrow \Omega$, then the $\Omega_{j}$ eventually swallow every compact subset of $\Omega$. However, they differ in what they require about the sets $\Omega_{j} \backslash \Omega$.

First I define a metric $\rho_{1}$ on bounded, nonempty, open sets via $\rho_{1}(U, V)=$ $\mathcal{H}(\bar{U}, \bar{V})+\mathcal{H}(\partial U, \partial V)$. If $\rho_{1}\left(\Omega_{j}, \Omega\right) \rightarrow 0$, then the $\Omega_{j}$ eventually swallow every compact subset of $\Omega$ and are eventually swallowed by every open neighborhood of $\bar{\Omega}$. The converse holds when $\Omega$ equals the interior of its closure, but not in general. By requiring that both the closures and the boundaries converge, convergence in the metric $\rho_{1}$ eliminates examples like the one above involving slits or punctures in the limit domain.

The metric $\rho_{1}$ can also be thought of in terms of functions. Define the distance function $d_{U}$ of an open set $U$ via $d_{U}(z)=\operatorname{dist}\left(z, \mathbf{C}^{n} \backslash U\right)$, where dist denotes the Euclidean distance. Then $\Omega_{j} \rightarrow \Omega$ according to the metric $\rho_{1}$ if and only if the continuous functions $d_{\Omega_{j}}$ converge uniformly on $\mathbf{C}^{n}$ to $d_{\Omega}$ and the $d_{\mathbf{C}^{n} \backslash \overline{\Omega_{j}}}$ converge uniformly to $d_{\mathbf{C}^{n} \backslash \bar{\Omega}}$.

In some contexts - one will appear below - it is useful to relax the hypothesis on how the sets $\Omega_{j} \backslash \Omega$ behave. They could be required to shrink in volume (Lebesgue $2 n$-dimensional measure), but not necessarily in terms of Euclidean distance from $\Omega$. I therefore introduce a second metric $\rho_{2}$ on bounded open sets via $\rho_{2}(U, V)=$ $\operatorname{vol}(U \backslash V)+\operatorname{vol}(V \backslash U)+\sup _{z \in \mathbf{C}^{n}}\left|d_{U}(z)-d_{V}(z)\right|$. Convergence of $\Omega_{j}$ to $\Omega$ in this metric allows $\Omega_{j}$ to have a long thin tail whose width shrinks to zero but whose length does not shrink.

So far I have not assumed that the open sets in question are connected. It is easy to see that the Bergman kernel function $K(w, z)$ for a disconnected open set is identically equal to zero if $w$ and $z$ are in different connected components, while if the points are in the same connected component, then $K(w, z)$ equals the Bergman kernel function of that component. I will say that a (possibly) disconnected, bounded, nonempty, open set is a $L u$ Qi-Keng open set if its Bergman kernel function has no zeroes when the two variables are in the same connected component.

Theorem. The Lu Qi-Keng open sets are nowhere dense in each of the following metric spaces, where the metric is $\rho_{1}$ :

(1) the bounded pseudoconvex open sets;

(2) the bounded connected pseudoconvex open sets (domains of holomorphy);

(3) the bounded strongly pseudoconvex open sets;

(4) the bounded connected strongly pseudoconvex open sets.

If one considers only open sets of Euclidean diameter less than some fixed constant $M$, then the same assertion holds when the metric is taken to be $\rho_{2}$.

I will base the proof of the theorem on the following two folklore lemmas. The ideas of the proofs are all in the literature, but since I do not know a reference for precisely these formulations, I will indicate proofs of the lemmas after the proof of the theorem.

Stability lemma for the Bergman kernel function. Let $\left\{\Omega_{j}\right\}$ be a sequence of bounded pseudoconvex open sets that converges, in the sense of either $\rho_{1}$ or $\rho_{2}$, to a nonempty bounded open set $\Omega$; in the case of $\rho_{2}$, assume also that the $\Omega_{j}$ have 
uniformly bounded diameters (this is automatic in the case of $\rho_{1}$ ). Suppose $U$ is a connected component of $\Omega$ that has $C^{\infty}$ regular boundary and that is separated from the rest of $\Omega$ (that is, the closure of $U$ is disjoint from the closure of $\Omega \backslash U$ ). Then the Bergman kernel functions of the $\Omega_{j}$ converge to the Bergman kernel function of $U$ uniformly on compact subsets of $U \times U$.

In the statement of the stability lemma, pseudoconvexity of the limit set $\Omega$ is automatic: the limit function $-\log d_{\Omega}$ inherits plurisubharmonicity from the functions $-\log d_{\Omega_{j}}$, which converge uniformly on compact subsets of $\Omega$.

A special case of considerable interest is when the $\Omega_{j}$ and $\Omega$ are all bounded, connected, pseudoconvex domains with $C^{\infty}$ regular boundaries. The lemma then says that if the $\Omega_{j}$ converge to $\Omega$, in the sense that $\Omega_{j}$ eventually swallows every compact subset of $\Omega$ and the volume of $\Omega_{j} \backslash \Omega$ tends to zero, then the Bergman kernel functions of the $\Omega_{j}$ converge uniformly on compact subsets of $\Omega \times \Omega$ to the Bergman kernel function of $\Omega$.

The $C^{\infty}$ regularity hypothesis in the lemma can be reduced to $C^{2}$ regularity, but I shall not prove this here.

I take the name of the second lemma from [10, Chap. 5, Exercise 21].

Barbell lemma. Suppose $G_{1}$ and $G_{2}$ are bounded, connected, strongly pseudoconvex domains in $\mathbf{C}^{n}$ with $C^{\infty}$ regular boundaries and with disjoint closures. Let $\gamma$ be a smooth curve (that is, a $C^{\infty}$ embedding of $[0,1]$ into $\mathbf{C}^{n}$ ) joining a boundary point of $G_{1}$ to a boundary point of $G_{2}$, and otherwise disjoint from the closures of $G_{1}$ and $G_{2}$, and let $V$ be an arbitrary neighborhood in $\mathbf{C}^{n}$ of the curve $\gamma$. Then there exists a bounded, connected, strongly pseudoconvex domain $\Omega$ with $C^{\infty}$ regular boundary such that $\Omega$ is contained in $G_{1} \cup G_{2} \cup V$, and $\Omega$ coincides with $G_{1} \cup G_{2}$ outside $V$.

When $G_{1}$ and $G_{2}$ are balls of equal size, and $\gamma$ is the shortest line segment joining them, then the domain $\Omega$ is a "barbell", or dumbbell.

The $C^{\infty}$ regularity can be changed everywhere in the statement of the lemma to $C^{k}$ regularity, where $k$ is any integer greater than or equal to 2 .

Proof of the theorem. I have not claimed that the bounded pseudoconvex open sets which fail to be Lu Qi-Keng form an open set in either of the metrics $\rho_{1}$ or $\rho_{2}$, and I do not know whether or not this is the case for sets with irregular boundaries. However, if $\Omega$ is, for example, a bounded strongly pseudoconvex open set with $C^{\infty}$ regular boundary, and the Bergman kernel function of $\Omega$ has zeroes on some connected component $\Omega_{0}$, then there is a $\rho_{1}$ neighborhood of $\Omega$ containing no pseudoconvex Lu Qi-Keng open set. Indeed, if a sequence of pseudoconvex open sets converges to $\Omega$ in the metric $\rho_{1}$, then the corresponding Bergman kernel functions converge on $\Omega_{0}$ to the Bergman kernel function of $\Omega_{0}$ by the stability lemma, and by Hurwitz's theorem these approximating Bergman kernel functions cannot all be zero-free on $\Omega_{0}$. The analogous statement holds for the metric $\rho_{2}$ if one restricts attention to sets of uniformly bounded Euclidean diameter.

Accordingly, to prove the theorem it will suffice to construct, arbitrarily close (according to either $\rho_{1}$ or $\rho_{2}$ ) to a given bounded pseudoconvex open set $G$, a bounded strongly pseudoconvex open set $\Omega$ with $C^{\infty}$ regular boundary whose Bergman kernel function does have zeroes on some connected component; if $G$ is connected, then $\Omega$ should be connected too. 
It is standard that the pseudoconvex open set $G$ can be exhausted from inside by strongly pseudoconvex open sets with $C^{\infty}$ regular boundaries: namely, by sublevel sets of a smooth, strictly plurisubharmonic exhaustion function. It is evident that these interior approximating sets converge to $G$ in both of the metrics $\rho_{1}$ and $\rho_{2}$. Consequently, there is no loss of generality in supposing from the start that $G$ is a bounded strongly pseudoconvex open set with $C^{\infty}$ regular boundary.

Place close to $G$ a strongly pseudoconvex domain $D$ with $C^{\infty}$ regular boundary and small diameter, the Bergman kernel function of $D$ having zeroes. (In $\mathbf{C}^{1}$, the domain $D$ could be an annulus; in higher dimensions, $D$ could be a small homothetic copy of the counterexample domain that I constructed in [1].) Then $G \cup D$ will be a disconnected strongly pseudoconvex open set that is close to $G$ in both of the metrics $\rho_{1}$ and $\rho_{2}$. This open set $G \cup D$ will serve as the required $\Omega$ to prove parts (1) and (3) of the theorem.

To prove parts (2) and (4) of the theorem, I need to produce a connected $\Omega$ when $G$ is connected. To do this, join $G$ to $D$ with a closed line segment $L$, and use the barbell lemma to construct a sequence of bounded, connected, strongly pseudoconvex open sets $\Omega_{k}$ with $C^{\infty}$ regular boundaries, the $\Omega_{k}$ being contained in $G \cup D \cup V_{k}$, where the $V_{k}$ are shrinking neighborhoods of the line segment $L$. The $\Omega_{k}$ converge to $G \cup D$ in the metric $\rho_{2}$, so the stability lemma and Hurwitz's theorem imply that the Bergman kernel function of $\Omega_{k}$ has zeroes (on $D$ ) when $k$ is sufficiently large. Since the Euclidean distance of $D \cup V_{k}$ from $G$ is small, $\Omega_{k}$ is close to $G$ in the metric $\rho_{1}$ as well as in the metric $\rho_{2}$. Thus one of the $\Omega_{k}$ serves as the required $\Omega$.

Proof of the stability lemma. The main point is to prove an $L^{2}$ approximation theorem for holomorphic functions. I claim that if $f$ is a square-integrable holomorphic function on $U$, and if a positive $\epsilon$ is prescribed, then for all sufficiently large $j$ there exists a square-integrable holomorphic function $g_{j}$ on $\Omega_{j}$ such that $\left\|f-g_{j}\right\|_{L^{2}\left(\Omega_{j} \cap U\right)}<\epsilon$ and $\left\|g_{j}\right\|_{L^{2}\left(\Omega_{j} \backslash U\right)}<\epsilon$.

I first need to show that the holomorphic functions in the Sobolev space $W^{1}(U)$ of square-integrable functions with square-integrable first derivatives are dense in the space of square-integrable holomorphic functions on $U$. This is a consequence of Kohn's global regularity theorem [9] for the $\bar{\partial}$-Neumann problem with weights. Namely, for a suitably large positive number $t$, the weighted $\bar{\partial}$-Neumann operator $N_{t}$ for $U$ is a bounded operator on the Sobolev space $W^{2}(U)$. Consequently, the corresponding weighted Bergman projection operator $P_{t}$, which satisfies the relation $P_{t}=\mathrm{Id}-\bar{\partial}_{t}^{*} N_{t} \bar{\partial}$, maps $W^{3}(U)$ into the holomorphic subspace of $W^{1}(U)$. Now if $f$ is a square-integrable holomorphic function in $U$, take a sequence $\left\{v_{j}\right\}$ of $C^{\infty}$ functions converging to $f$ in $L^{2}(U)$, and project these functions by $P_{t}$. The functions $P_{t} v_{j}$ are holomorphic functions in $W^{1}(U)$ that converge to $f$ in $L^{2}(U)$.

Therefore, there is no loss of generality in assuming from the start that the holomorphic function $f$ lies in $W^{1}(U)$. Consequently, $f$ is the restriction to $U$ of a function $F \in W^{1}\left(\mathbf{C}^{n}\right)$.

It follows from the hypothesis of the lemma that there is an open neighborhood $V$ of the closure of $U$ such that the $2 n$-dimensional Lebesgue measure of $V \cap\left(\Omega_{j} \backslash U\right)$ tends to zero as $j \rightarrow \infty$. There is no harm in cutting off the function $F$ so that its support lies inside $V$.

The one-form $\bar{\partial} F$ is then defined on all of $\mathbf{C}^{n}$, zero on $U$, zero outside $V$, and square-integrable. Since the measure of $V \cap\left(\Omega_{j} \backslash U\right)$ shrinks to zero, the $L^{2}\left(\Omega_{j}\right)$ norm 
of $\bar{\partial} F$ tends to zero as $j \rightarrow \infty$. Use Hörmander's $L^{2}$ theory [7] to solve the equation $\bar{\partial} u_{j}=\bar{\partial} F$ on $\Omega_{j}$ for a square-integrable function $u_{j}$ whose $L^{2}\left(\Omega_{j}\right)$ norm is bounded by a constant (depending only on the uniform bound on the diameters of the $\Omega_{j}$ ) times the $L^{2}\left(\Omega_{j}\right)$ norm of $\bar{\partial} F$. Thus the norm of $u_{j}$ on $\Omega_{j}$ tends to zero as $j \rightarrow \infty$. Consequently, the function $g_{j}:=F-u_{j}$, which is holomorphic and square-integrable on $\Omega_{j}$, has norm on $\Omega_{j} \cap U$ close to the norm of $f$ when $j$ is large. Also, the norm of $g_{j}$ on $\Omega_{j} \backslash U$ tends to zero with the measure of $V \cap\left(\Omega_{j} \backslash U\right)$. This confirms the claimed approximation property.

The remainder of the proof of the stability lemma follows standard lines. However, I mention that I am dispensing with the hypothesis of monotonicity of the domains that is typically assumed [8, pp. 180-182], [15, pp. 36-39].

Fix a point $z$ in $U$. The Bergman kernel function $K(\cdot, z)$ (for $U$, or equivalently for $\Omega$ when the free variable is in $U$ ) is the unique square-integrable holomorphic function $f$ on $U$ that maximizes $f(z)$ subject to the nonlinear constraint $f(z) \geq$ $\|f\|_{L^{2}(U)}^{2}$. Let $f_{j}$ denote the corresponding extremal function for the approximating domain $\Omega_{j}$. By the mean-value property of holomorphic functions, $f_{j}(z)$ is bounded by a constant times $\left\|f_{j}\right\|_{L^{2}\left(\Omega_{j}\right)}$ times an inverse power of the distance from $z$ to the boundary of $\Omega_{j}$; the extremal property of $f_{j}$ then implies that $\left\|f_{j}\right\|_{L^{2}\left(\Omega_{j}\right)}$ too is bounded by a constant times an inverse power of the distance from $z$ to the boundary of $\Omega_{j}$. Therefore the $\left\|f_{j}\right\|_{L^{2}\left(\Omega_{j}\right)}$ are uniformly bounded, and so the $f_{j}$ form a normal family on $U$. Consequently, the $f_{j}$ have a subsequence that converges uniformly on compact subsets of $U$ to a holomorphic limit function $f_{\infty}$. (Once I show that the limit $f_{\infty}$ actually is $f$, it will follow that the original sequence $\left\{f_{j}\right\}$, not just a subsequence, converges to $f$.)

By Fatou's lemma, it follows that the limit function $f_{\infty}$ satisfies $f_{\infty}(z) \geq$ $\left\|f_{\infty}\right\|_{L^{2}(U)}^{2}$. By the approximation property proved above, there exists a squareintegrable holomorphic function $g_{j}$ on $\Omega_{j}$ such that $g_{j}(z) \geq\left\|g_{j}\right\|_{L^{2}\left(\Omega_{j}\right)}^{2}$, and $g_{j}(z) \geq$ $\left(1-\delta_{j}\right) f(z)$, where the positive numbers $\delta_{j}$ tend to zero as $j \rightarrow \infty$. The extremal function $f_{j}$ therefore has the property that $f_{j}(z) \geq\left(1-\delta_{j}\right) f(z)$. Consequently, $f_{\infty}(z) \geq f(z)$. The uniqueness of the extremal function implies that $f_{\infty}=f$. This proves that the Bergman kernel functions $K_{j}(w, z)$ for the $\Omega_{j}$ converge pointwise to $K(w, z)$ on $U \times U$.

Since $\left|K_{j}(w, z)\right|^{2} \leq K_{j}(w, w) K_{j}(z, z)$ by the Cauchy-Schwarz inequality, and the right-hand side is bounded by a constant depending only on the distances of $z$ and $w$ from the boundary of $\Omega_{j}$, the functions $K_{j}(\cdot, \cdot)$ form a normal family in $U \times U$. From the normality and the pointwise convergence just proved, it is immediate that the convergence is uniform on compact subsets of $U \times U$.

Proof of the barbell lemma. In the complex plane $\mathbf{C}^{1}$, there is nothing to prove, for every planar domain is strongly pseudoconvex. In higher dimensions, there is no loss of generality in supposing that the curve $\gamma$ meets the boundaries of $G_{1}$ and $G_{2}$ transversely, since the barbell $\Omega$ is not prescribed inside the neighborhood $V$. By [4, Theorem 4] (a result that the authors attribute to [5]), the set $\bar{G}_{1} \cup \bar{G}_{2} \cup \gamma$ has a basis of Stein neighborhoods, so there exists a connected, strongly pseudoconvex domain with $C^{\infty}$ regular boundary that outside $V$ is a small perturbation of $G_{1} \cup G_{2}$. This conclusion is already enough for the application to the proof of the main theorem.

The stronger statement that one can find a barbell that actually matches $G_{1} \cup G_{2}$ outside a neighborhood of the curve $\gamma$ was demonstrated by Shcherbina for the 
case when $G_{1}$ and $G_{2}$ are balls [14, Lemma 1.2 and its Corollary]. The general case follows from this special one because any strongly pseudoconvex domain can be perturbed in an arbitrarily small neighborhood of a boundary point to obtain a new strongly pseudoconvex domain whose boundary near that point is a piece of the boundary of a ball. This can be seen from the patching lemma for strictly plurisubharmonic functions in [3, Lemma 3.2.2] by taking the totally real set there to be a single point.

\section{OPEN QUESTIONS}

(1) In the stability lemma, the $C^{\infty}$ regularity hypothesis can be reduced to $C^{2}$ regularity by inspecting Kohn's proof [9] to see that $C^{k+1}$ boundary regularity suffices for $W^{k}$ regularity of the weighted $\bar{\partial}$-Neumann operator; one also needs techniques as in [2] to see that the weighted Bergman projection has the same regularity as the weighted $\bar{\partial}$-Neumann operator. Can the hypothesis in the stability lemma be reduced to $C^{1}$ boundary regularity?

(2) The conclusion of the theorem - that most pseudoconvex domains are not $\mathrm{Lu}$ Qi-Keng domains - changes if the topology on domains is changed. For example, any small $C^{\infty}$ perturbation of the unit ball is a Lu Qi-Keng domain [6]. Does the set of bounded pseudoconvex Lu Qi-Keng domains with $C^{1}$ regular boundary have nonempty interior in the $C^{1}$ topology on pseudoconvex domains? This is the case for domains in the complex plane $\mathbf{C}^{1}$.

(3) My proof of the stability lemma for the Bergman kernel function uses pseudoconvexity. Can the word "pseudoconvex" be removed from the statement of the main theorem?

(4) Is every bounded convex domain a Lu Qi-Keng domain?

\section{REFERENCES}

[1] H. P. Boas, Counterexample to the Lu Qi-Keng conjecture, Proc. Amer. Math. Soc. 97 (1986), 374-375. MR 87i:32035

[2] H. P. Boas and E. J. Straube, Equivalence of regularity for the Bergman projection and the $\bar{\partial}$-Neumann operator, manuscripta math. 67 (1990), 25-33. MR 90k:32057

[3] Yakov Eliashberg, Topological characterization of Stein manifolds of dimension $>2$, International J. Math. 1 (1990), no. 1, 29-46. MR 91k:32012

[4] John E. Fornæss and Bill Zame, Runge exhaustions of domains in $\mathbf{C}^{n}$, Math. Z. 194 (1987), 1-5. MR 88c:32026

[5] John Erik Fornæss and Edgar Lee Stout, Spreading polydiscs on complex manifolds, Amer. J. Math. 99 (1977), no. 5, 933-960. MR 57:10009

[6] R. E. Greene and Steven G. Krantz, Stability properties of the Bergman kernel and curvature properties of bounded domains, in Recent Developments in Several Complex Variables, Princeton Univ. Press, Princeton, NJ, 1981, 179-198. MR 83d:32023

[7] Lars Hörmander, $L^{2}$ estimates and existence theorems for the $\bar{\partial}$-operator, Acta Math. 113 (1965) 89-152. MR 31:3691

[8] M. Jarnicki and P. Pflug, Invariant Distances and Metrics in Complex Analysis, de Gruyter, Berlin, New York, 1993. MR 94k:32039

[9] J. J. Kohn, Global regularity for $\bar{\partial}$ on weakly pseudo-convex manifolds, Trans. Amer. Math. Soc. 181 (1973), 273-292. MR 49:9442

[10] Steven G. Krantz, Function Theory of Several Complex Variables, second edition, Wadsworth \& Brooks/Cole, Pacific Grove, CA, 1992. MR 93c:32001

[11] Lu Qi-Keng, On Kaehler manifolds with constant curvature, Chinese Math. 8 (1966), 283298; English translation of Acta Math. Sinica 16 (1966), 269-281. MR 34:6806

[12] I. Ramadanov, Sur une propriété de la fonction de Bergman, C. R. Acad. Bulgare Sci. 20 (1967), 759-762. MR 37:1632 
[13] I.-P. Ramadanov, Some applications of the Bergman kernel to geometrical theory of functions, Complex Analysis, Banach Center Publications, Vol. 11, PWN-Polish Scientific Publishers, Warsaw, 1983, 275-286. MR 85h:32040

[14] N. V. Shcherbina, On fibering into analytic curves of the common boundary of two domains of holomorphy, Math. USSR Izvestiya 21 (1983), no. 2, 399-413; English translation of Izv. Akad. Nauk SSSR Ser. Mat. 46 (1982), no. 5, 1106-1123. MR 84b:32018

[15] M. Skwarczyński, Biholomorphic invariants related to the Bergman function, Dissertationes Math. 173 (1980). MR 82e:32038

[16] N. Suita and A. Yamada, On the Lu Qi-Keng conjecture, Proc. Amer. Math. Soc. 59 (1976), 222-224. MR 54:13142

Department of Mathematics, Texas A\&M University, College Station, Texas 77843 3368

E-mail address: boas@math.tamu.edu 